\title{
A viscosity-dependent affinity sensor for continuous monitoring of glucose in biological fluids
}

\author{
Christophe Boss ${ }^{\mathrm{a}, *}$, Eric Meurville ${ }^{\mathrm{a}}$, Jean-Michel Sallese ${ }^{\mathrm{b}}$, Peter Ryser ${ }^{\mathrm{a}}$ \\ ${ }^{a}$ Laboratory of Microengineering for Manufacturing, Ecole Polytechnique Fédérale de Lausanne, Station 17, 1015 Lausanne, Switzerland \\ ${ }^{\mathrm{b}}$ Institute of Electrical Engineering, Ecole Polytechnique Fédérale de Lausanne, Station 11, 1015 Lausanne, Switzerland
}

\section{A R T I C L E I N F O}

\section{Article history:}

Received 27 July 2011

Received in revised form 5 September 2011

Accepted 15 September 2011

Available online 22 September 2011

\section{Keywords:}

Glucose sensing

Affinity sensor

Micromechanical sensor

Viscosity

Concanavalin A

Dextran

\begin{abstract}
A B S T R A C T
We present a viscometric affinity biosensor for continuous monitoring of glucose in biological fluids such as blood and plasma. The sensing principle of this chemico-mechanical sensor is based upon the viscosity variation of a sensitive fluid with glucose concentration. Basically, this device includes both an actuating and a sensing piezoelectric diaphragms as well as a flow-resistive microchannel. In order to confine the sensitive fluid and allow glucose diffusion into the sensor, a free-standing alumina nanoporous membrane is also used as size-selective interface. Measurements carried out at nominal temperatures of 25 and $37^{\circ} \mathrm{C}$ reveal that this sensor topology exhibits a high resolution in the current range of physiological blood glucose concentrations, i.e. $2-20 \mathrm{mM}$. In addition, complete reversibility was also demonstrated for at least 3 days. Finally, measurements performed in human blood serum confirm that this sensor fulfils all basic requirements for a use in continuous glucose monitoring of biological fluids.
\end{abstract}

(c) 2011 Elsevier B.V. All rights reserved.

\section{Introduction}

A compact, reliable and affordable continuous glucose monitoring system is of great interest for medical applications and would allow a better diabetes management compared to current finger pricking (Klonoff, 2005). A strict glycemic control would also improve clinical outcomes for critically ill patients in intensive care units, impending glycemic excursions (Krinsley, 2004).

Today, existing continuous glucose monitoring devices are lacking accuracy, stability and robustness, and consequently are not used in the intensive care units. Moreover, diabetic patients still have to prick their finger to get precise measurement and calibrate existing devices.

Presently, glucose detection methods are based on electrochemical techniques that are hardly compatible with in vivo monitoring. These electrochemical measurements are affected by biofouling (the adhesion of biomolecules to surfaces) that degrades the sensitivity and reproducibility, thus requiring frequent calibrations (Wang, 2008). Furthermore, unwanted electrochemically active molecules may interfere with the metabolite of interest, resulting in a lack of reliability (Heller and Feldman, 2008).

An alternative approach to overcome these limitations is affinity sensing. Affinity sensing is more tolerant to biofouling that only

\footnotetext{
* Corresponding author. Tel.: +41 216937 780; fax: +41 216933891.

E-mail address: christophe.boss@epfl.ch (C. Boss).
}

increases the time for the system to stabilize, and is also insensitive to electroactive interferences. For these reasons, investigation on affinity binding sensors has been undertaken using various technologies, such as fluorescence (Ballerstadt et al., 2004, 2006, 2007; Ballerstadt and Schultz, 2000) or viscosity-based (Beyer et al., 2001; Diem et al., 2004; Huang et al., 2009; Kuenzi et al., 2011; Zhao et al., 2007) principle. Recently, hydrogel-based sensors have emerged as promising tools for affinity sensing (Tierney et al., 2009a, 2009b). However, the development of biosensors for medical and biological applications that allows for long-term reversibility and stability remains a challenge.

In this context, we propose a novel chemico-mechanical concept which aims at detecting viscosity changes of a solution featuring a selective affinity for glucose. In this paper, we present a viscosity sensing principle based on an induced liquid flow in a microchannel upon piezoelectric diaphragm actuation. Both a theoretical model of the sensor and extensive characterizations in isotonic saline solution and human blood serum are presented.

\section{Sensing principle}

\subsection{Glucose-dependent viscosity of the sensitive fluid}

The sensitive fluid used within the sensor is the key element for glucose detection. This is based on the competitive affinity of two saccharides, namely glucose and dextran, to a specific saccharidebinding protein, Concanavalin A (ConA). The sensing principle was 
a

Low glucose

concentration

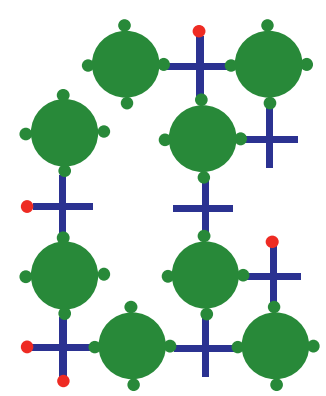

High viscosity
High glucose

concentration

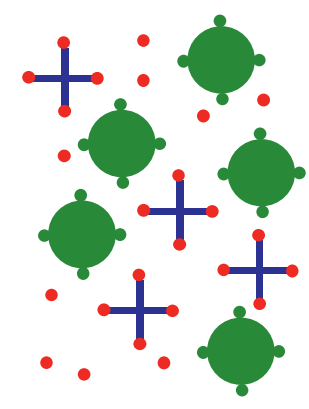

Low viscosity
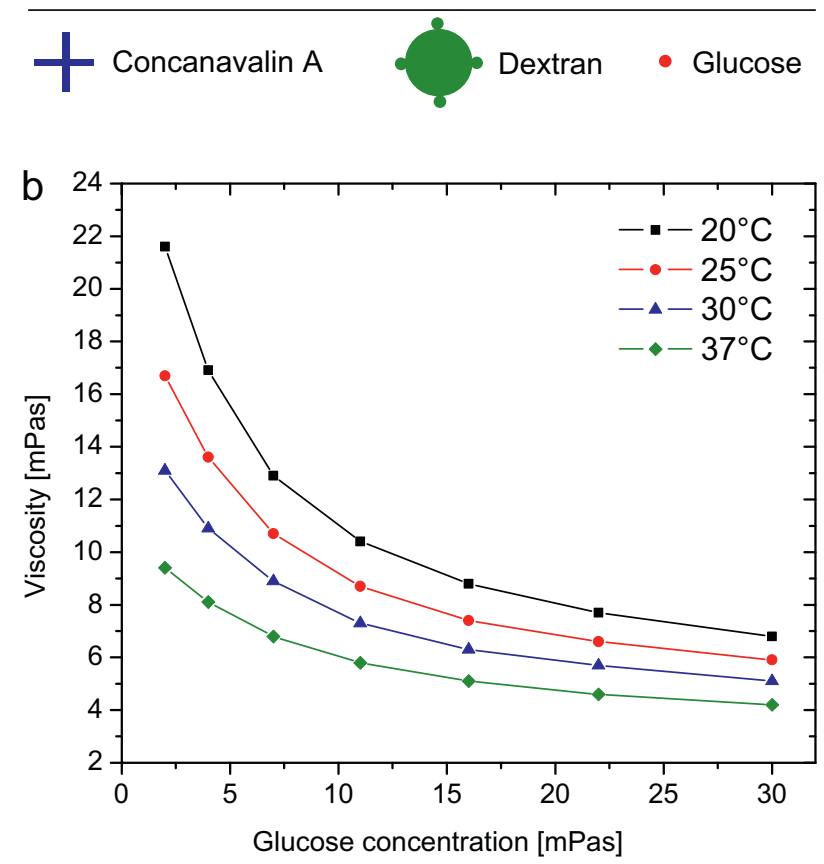

Fig. 1. (a) Principle of the glucose sensitive fluid: competitive binding of glucose and dextran on ConA in the case of low and high glucose concentrations. (b) Characteristics of the low viscosity sensitive fluid used within the sensor.

first demonstrated by optical measurement of glucose concentration using fluorescence-labelled dextran (Schultz et al., 1982), and then from the change in the viscosity of an aqueous blend of dextran and ConA (Ballerstadt and Ehwald, 1994). This competitive affinity process was shown to be reversible and highly sensitive.

This viscosity variation of a sensitive fluid using a highmolecular-weight dextran is eventually the core of our sensor. At low glucose concentration, dextran molecules are cross-linked by binding to ConA, forming a viscous solution (Fig. 1a). Next, when the glucose concentration increases, dextran molecules are partially replaced by glucose ones at the binding sites of ConA. As a result, the network ConA-dextran is weakened and the viscosity of the sensing solution decreases.

The viscosity variations are especially strong in the human glycemic range (2-30 mM glucose). Indeed, changes in viscosity as high as one order of magnitude in the physiological relevant range were reported (Kuenzi et al., 2010). The sensitive fluid viscosity can also be tailored for the sensor of interest by changing its composition in terms of dextran and ConA. We chose a low viscosity sensitive fluid, which was characterized using a standard capillary viscometer (Fig. 1b). It is worth noticing that the sensitivity of the fluid is particularly high in the hypoglycaemic range (2-4 mM), whereas it decreases in the hyperglycaemic range (7-30 mM). High accuracy in hypoglycaemic region is mandatory to make this sensitive fluid particularly well suited for patient monitoring. Beside that, the sensitive fluid viscosity is highly temperature dependent. When raising the temperature from 25 to $37^{\circ} \mathrm{C}$, the sensitive fluid viscosity decreases on average by $38 \%$. This dependance should therefore to be taken into account through calibration when working in physiological conditions.

\subsection{Principle of piezoelectric-based viscosity measurements}

Basically, viscosity is extracted from the relationship between pressure and flow when the sensitive solution goes through a channel. In our experiment, the sensor comprises two microchambers filled with the sensitive fluid that communicate through a microchannel and a rigid nanoporous semi-permeable membrane (Fig. 2a). This membrane has two roles: it confines the sensitive fluid inside the sensor (large molecules cannot go through) and ensures that the glucose can pass through, meaning that the concentration in the sensor and in the liquid should be the same. Each chamber is composed of a flexible piezoelectric diaphragm which whether deflects under voltage (actuating diaphragm) or generates a voltage under some pressure load (sensing diaphragm).

These actuating and sensing piezoelectric diaphragms together with the microchannel are the basis for viscosity detection. When a voltage is applied on the actuating piezoelectric diaphragm, the latter deflects and tends to generate a flow through the microchannel. Due to its small section, the microchannel exhibits a resistance to the flow which depends on the fluid viscosity. Next, the flow through the microchannel is recorded using the voltage induced by the sensing piezoelectric deflection.

Both transient and harmonic electric signals can be used to actuate the diaphragm (Fig. 2b). The way viscosity is measured will depend on these wave forms. In transient mode, a constant voltage is suddenly applied to the actuating diaphragm for a given time, and then it is switched off. These states generate a strain in the piezoelectric membrane that in turn will create some pressure in the fluid. Due to its small cross section, the microchannel exhibits a

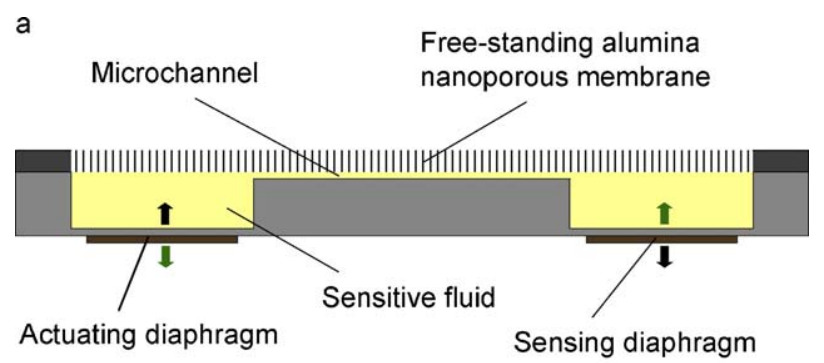

b Transient mode Harmonic mode

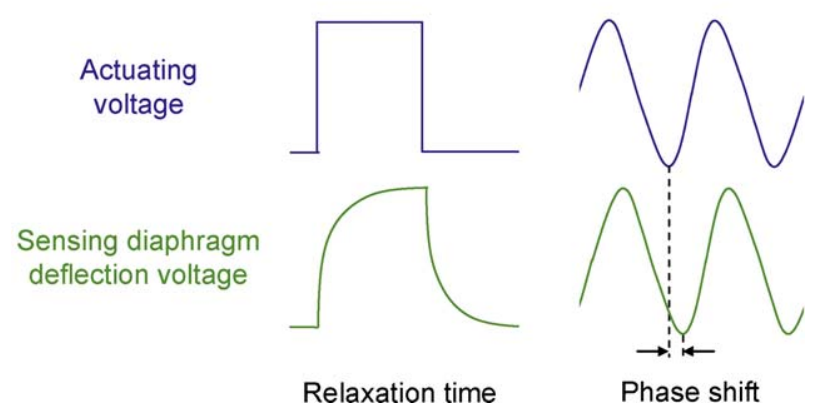

Fig. 2. Schematic cross view of the glucose sensor (a) and description of the measurement methods for viscosity detection (b). 
resistance to the flow which depends upon the viscosity of the sensitive fluid. The system is characterized by a relaxation time that depends upon the viscosity of the sensitive fluid: the more viscous the sensitive fluid, the larger the relaxation time.

Conversely, under harmonic operation, a sinusoidal voltage is applied on the actuating diaphragm. This generates an alternative flow inside the microchannel, which in turn generates a sinusoidal deflection and induced voltage of the sensing diaphragm. Since the amplitude and the phase shift between the applied and induced piezoelectric voltages characterizes the viscosity, we have a simple tool to assess the fluid characteristics: the more viscous the sensitive fluid, the smaller the deflection and the larger the phase shift.

\subsection{Sensor modelling}

Modelling aspects of the device are quite important to optimize the sensor with respect to the expected variation of the sensitive fluid viscosity. A sketch of the sensor principle used for the derivation of the generic analytical solution is explained in Fig. 3.

Under quasi static conditions, the laminar viscous flow of a liquid through a circular channel with a length much greater than its diameter, is described by the Hagen-Poiseuille equation

$Q=\frac{\pi D^{4}}{128 \eta L} \Delta P$

where $Q$ is the volumetric flow rate, $D$ and $L$ are, respectively, the channel diameter and length, $\eta$ is the liquid viscosity and $\Delta P$ represents the pressure drop across the channel.

On the other hand, the deflection of the center of a piezoelectric diaphragm depends on the applied voltage and pressure load. Assuming small deflections, the relationship is linear and given by

$x=\alpha V-\beta P$

where $x$ is the deflection of the piezoelectric diaphragm center, $V$ the voltage applied to the piezoelectric diaphragm and $P$ the pressure acting against the diaphragm deflection. The coefficients $\alpha$ and $\beta$ depend on the diaphragm geometry and material properties. For static deflections of a circular multi-layered piezoelectric diaphragm, their values are obtained from the model of Deshpande and Saggere (2007). As no external voltage is applied to the sensing piezoelectric diaphragm, its deflection only depends on the pressure load:

$x_{2}=\beta_{2} P_{2}$

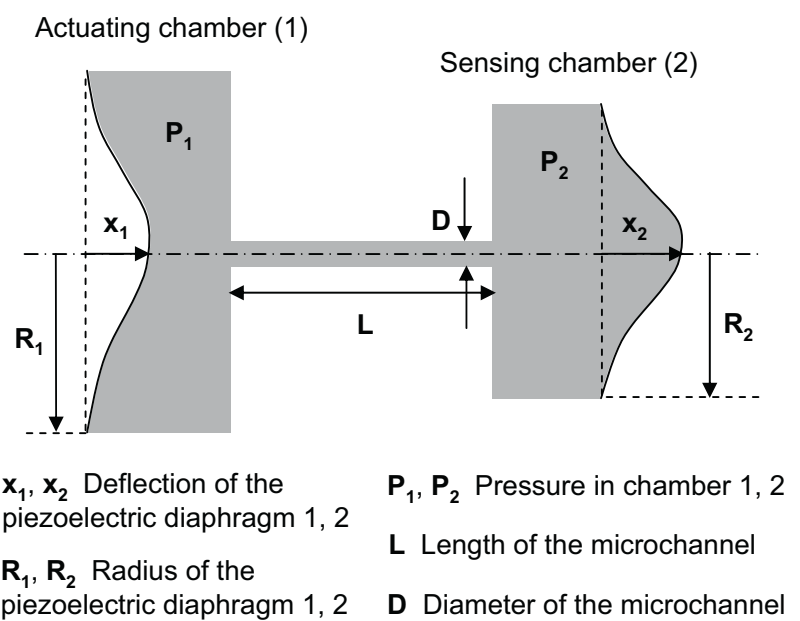

Fig. 3. Schematic representation of the viscosity sensing principle with the used notations for the theoretical calculations.
Next, the total volume displaced by a fixed-edge circular diaphragm under uniform load is given by

$V=\frac{1}{3} x \pi R^{2}$

where $x$ and $R$ are, respectively, the deflection of the center and the radius of the diaphragm (note that this equation was derived for a diaphragm under uniform pressure load and not for a multi-layered piezoelectric diaphragm, but we will nevertheless use it as a good approximation).

The volumetric flow rate through the channel is equal to the volume change under the sensing piezoelectric diaphragm:

$Q=\frac{d V}{d t}=\frac{\pi R_{2}^{2}}{3} \frac{d x}{d t}$

Coupling the Hagen-Poiseuille flow through the channel with the piezoelectric diaphragms deflections (Eqs. (1)-(5)), and considering a sinusoidal voltage $V_{1}=V_{1}^{0} \sin (\omega t)$ for actuation, we get the time dependent differential equation:

$\frac{128 \eta L R_{2}^{2} \beta_{1}}{3 D^{4}} \frac{d x_{2}}{d t}+\left(\frac{R_{2}^{2} \beta_{2}+R_{1}^{2} \beta_{1}}{R_{1}^{2} \beta_{2}}\right) x_{2}=\alpha_{1} V_{1}^{0} \sin (\omega t)$

The solution of the differential equation (Eq. (6)) is composed of a transient and a stationary part. Here, we are mainly interested in the stationary solution describing the deflection of the sensing piezoelectric diaphragm which is given by:

$x_{2}(t)=A \sin (\omega t-\varphi)$

with

$A=\frac{R_{1}^{2} \beta_{2}}{R_{2}^{2} \beta_{2}+R_{1}^{2} \beta_{1}} \frac{\alpha_{1} V_{1}^{0}}{\sqrt{\tau^{2} \omega^{2}+1}}$

and

$\varphi=\arctan (\tau \omega)$

where

$\tau=\frac{128 L}{3 D^{4}} \frac{R_{2}^{2} R_{1}^{2} \beta_{2} \beta_{1}}{R_{2}^{2} \beta_{2}+R_{1}^{2} \beta_{1}} \eta$

is the relaxation time of the system.

This means that when a sinusoidal voltage is applied to the actuating piezoelectric diaphragm, the sensing diaphragm will also deflect harmonically, but its amplitude and phase shift will now depend on the relaxation time of the system which is found to be proportional to the liquid viscosity (in addition to the geometric and piezoelectric diaphragms characteristics). Next, these mechanical signals will generate electrical waveforms that will be used to extract the viscosity of the sensitive fluid.

Aside this analysis, since a time dependent sinusoidal voltage is applied to the actuating diaphragm, we can wonder if the flow can still be considered in steady state. When a high viscous liquid pulsates slowly in a narrow pipe, the instantaneous velocity distribution is given by a parabola as in the case of steady Poiseuille flow. Uchida (1956) demonstrated that the steady state criteria was still justified provided $\sqrt{\omega / v} D \ll 1$, where $\omega$ is the pulsation of the flow, $v$ the kinematic viscosity and $D$ the diameter of the channel. As the sensitive liquid viscosity is several times that of the water and since the channel is only some micrometers diameter, using $\omega=10 \mathrm{rad} / \mathrm{s}$, $v=70 \mathrm{~mm}^{2} / \mathrm{s}$ and $D=100 \times 10^{-6} \mathrm{~m}$, we find a typical value for the steady state criteria equal to $4.24 \times 10^{-2}$. Therefore, the quasisteady condition is satisfied and the Hagen-Poiseuille equation can be used. 


\section{Experimental}

\subsection{Sensitive fluid characteristics}

The sensitive fluid was prepared following Kuenzi et al. (2010). It consists of $2 \%[\mathrm{w} / \mathrm{w}]$ dextran 3200 (PSS) and $0.4 \%[\mathrm{w} / \mathrm{w}]$ ConA (Sigma) in a buffered saline solution (10 $\mathrm{mM}$ Tris base, $1 \mathrm{mM} \mathrm{CaCl}_{2}$, $1 \mathrm{mM} \mathrm{MnCl} 2,0.05 \% \mathrm{NaN}_{3}, 0.15 \mathrm{M} \mathrm{NaCl}$ ). Two metal ions, $\mathrm{Mn}^{2+}$ and $\mathrm{Ca}^{2+}$, were added to the solution to activate the glucose binding site of ConA. This conformational change strongly increases its affinity to glucose. The $\mathrm{pH}$ was adjusted to the physiological value $(\mathrm{pH}$ 7.4), which also ensures a maximal proportion of tetrameric ConA able to bind up to 4 glucose groups and form a large network. The saline concentration was set to its physiological value $(0.15 \mathrm{M} \mathrm{NaCl})$ and $\mathrm{NaN}_{3}$ was added as preservative. The viscosity of the sensitive fluid was characterized using a capillary viscometer. Its values were ranging from 5.9 to $16.7 \mathrm{mPa} \mathrm{s}$ at $25^{\circ} \mathrm{C}$ and from 4.2 to $9.4 \mathrm{mPa}$ s at $37^{\circ} \mathrm{C}$ (Fig. 1b).

\subsection{Sensor architecture}

The sensor was fabricated by stereolithography using a biocompatible medical-grade resin (Proform). The actuating diaphragm was a $3 \mathrm{~mm}$ diameter, $50 \mu \mathrm{m}$ thick disc of lead zirconate titanate (PZT) (Audiowell Electronics) glued onto a $10 \mu \mathrm{m}$ thick brass foil (Goodfellow). On the other hand, the sensing diaphragm was a $3 \mathrm{~mm}$ diameter, $28 \mu \mathrm{m}$ thick disc of polyvinylidene fluoride (PVDF) (Measurement Specialties) glued onto a $10 \mu \mathrm{m}$ thick brass foil. The semi-permeable membrane was a $50 \mu \mathrm{m}$ thick alumina nanoporous membrane with $2-4 \mathrm{~nm}$ diameter pores (Synkera Technologies). A $100 \mu \mathrm{m} \times 100 \mu \mathrm{m}$ section glass capillary was used for the microchannel (Vitrocom). The sensor assembly was realized using medical-grade adhesive epoxy (Loctite M-21HP). Finally, the sensor was $200 \mu \mathrm{m}$ thick and contained $4 \mu \mathrm{L}$ of sensitive fluid.

\subsection{In vitro sensor test system}

As the viscosity of the sensitive fluid is temperature-dependent, the whole setup was located in a thermally regulated enclosure $\left( \pm 0.01^{\circ} \mathrm{C}\right)$. The sinusoidal voltage generation and acquisition were performed using a multifunction DAQ board PCI-6052E from National Instrument. The actuating piezoelectric diaphragm was driven with a low-frequency sinusoidal voltage $(1-5 \mathrm{~Hz})$ amplified up to $30 \mathrm{~V}$ by means of a high voltage amplifier (WMA-02 from Falco Systems). The sensing voltage was amplified using an instrumentation amplifier (AD8661 from Analog Device). The voltage generation, acquisition and phase shift computation were performed using a LabVIEW interface.

To evaluate the characteristics and response of the device, glucose measurements were performed in isotonic saline solution (10 mM Tris base, $1 \mathrm{mM} \mathrm{CaCl}_{2}, 1 \mathrm{mM} \mathrm{MnCl} 2,0.05 \% \mathrm{NaN}_{3}, 0.15 \mathrm{M}$ $\mathrm{NaCl}$ ). Glucose solutions of varying concentrations were pumped into the test cell using a computer controlled syringe pump (Tecan Cavro XCalibur). Subsequently, the device was also evaluated using human blood serum as a model biological fluid. The human serum was stored at $-20^{\circ} \mathrm{C}$. Before use, $0.1 \%$ sodium azide $\left(\mathrm{NaN}_{3}\right)$ was added as preservative, then the human serum was filtered (Millex HV $0.45 \mu \mathrm{m}$ syringe filter) to remove aggregates which may clog the semi-permeable membrane. Glucose concentration in the human serum was measured using a standard glucose meter (Accu-Chek). Concentrated (2 M) D-glucose solutions were added to the human serum to achieve high glucose levels.

Since a high voltage is not compatible for in vivo applications, we can wonder whether a lower actuating voltage could also be used. A flow through the whole capillary is required to equilibrate the glucose concentration inside the capillary. A voltage of $30 \mathrm{~V}$ was imposed by the sensor design; it produces a bending of $2.7 \mu \mathrm{m}$ of the diaphragm center, which generates a flow over $1.2 \mathrm{~mm}$ in the $100 \mu \mathrm{m} \times 100 \mu \mathrm{m}$ section glass capillary. The voltage could be reduced by decreasing the microchannel dimensions (e.g. a voltage of $5 \mathrm{~V}$ could be used with a $500 \mu \mathrm{m}$ length, $50 \mu \mathrm{m} \times 50 \mu \mathrm{m}$ section microchannel). Other alternatives could include the reduction of the piezoelectric layer thickness, as an equivalent electric field would be produced with a lower voltage, or using a microchannel with a porous side wall, which would allow the glucose diffusion into the microchannel.

\section{Results and discussion}

\subsection{Sensor characterization at various temperatures}

The sensor was characterized at 25 and $37^{\circ} \mathrm{C}$. Stepwise increasing levels of glucose concentrations (2-4-7-11-16 mM) have been used, followed by similar decreasing glucose concentrations (16-11-7-4-2 mM) (Fig. 4a). The data indicate that the sensing principle is highly reversible; the phase shift characterizing a glucose level is the same after increasing or decreasing the glucose concentration.

The reversibility of the sensitive solution was expected since the reversibility of the ConA-saccharide binding was previously
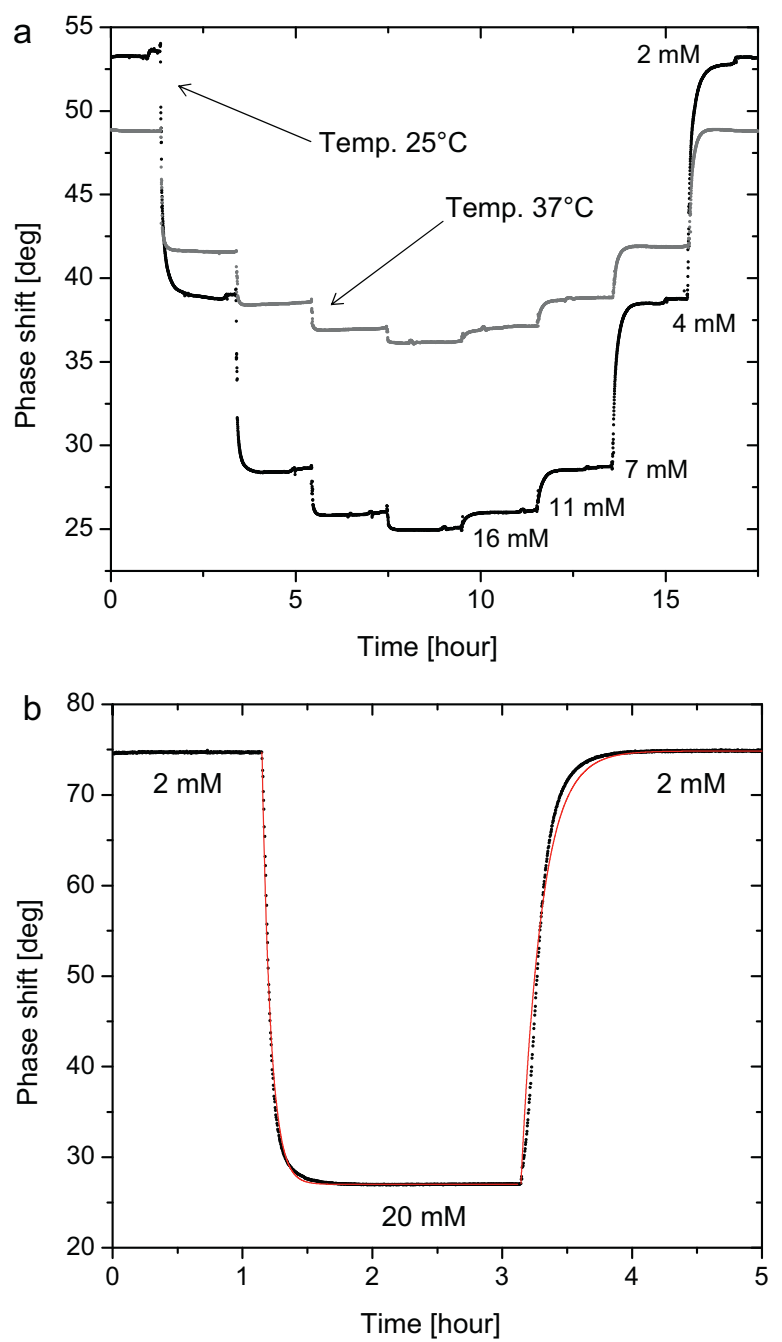

Fig. 4. (a) Phase shift response to stepwise increasing and decreasing glucose concentrations $(2,4,7,11,16 \mathrm{mM})$ in isotonic saline solution at 25 and $37^{\circ} \mathrm{C}$. (b) Sensor response to increasing and decreasing glucose concentrations fitted with an exponential model. 


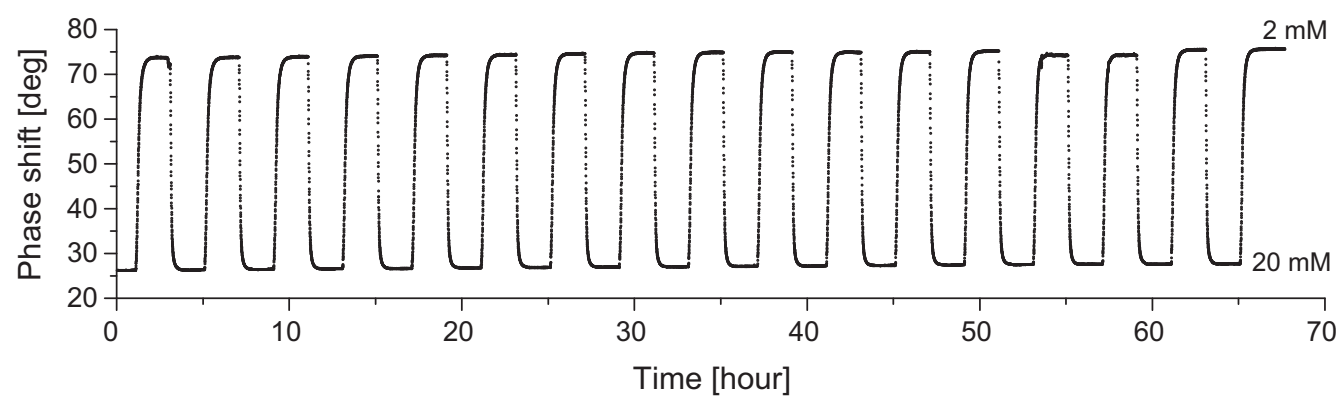

Fig. 5. Phase shift response to 17 full cycles of glucose concentrations $(2 \mathrm{mM}$ and $20 \mathrm{mM})$ in isotonic saline solution at $25^{\circ} \mathrm{C}$.

demonstrated. Nevertheless, the difficulty is the confinement of the sensitive fluid inside the sensor, given that ConA is a small protein. The ConA hydrodynamic radius in tetramer configuration (highly prevailing at $\mathrm{pH} 7.4$ ) is $4.4 \mathrm{~nm}$ whereas the nanoporous alumina membrane pores distribution was $2-4 \mathrm{~nm}$. The high degree of reversibility obtained implies that ConA molecules in tetramer configuration were well confined by the alumina membrane. Moreover, the response time of the sensor did not increase with time, indicating that dextran and ConA did not clog the pores. The sensing principle worked well, glucose diffusing freely in and out of the sensor through the nanoporous membrane, whereas the sensitive fluid was completely confined inside the sensor.

Finally, we observe that the degree of glucose-induced phase shift change is reduced by about $55 \%$ when the temperature increases. This was expected, since the sensitive fluid viscosity is highly temperature dependent. However, the reduced sensitivity at $37^{\circ} \mathrm{C}$ does not represent a practical limitation for the detection of the glucose concentration provided some calibrations are carried out.

\subsection{Sensor response dynamics}

The diffusion of glucose in-outside of the sensor was well described by an exponential model, which tends to show that diffusion processes are governing the sensor kinetics (Fig. 4b). The response time was determined by fitting the sensor response to multiple glucose variations (20 measurements) using an exponential relationship. At $37^{\circ} \mathrm{C}$, the time response of the sensor (time to reach $90 \%$ of the equilibrium value) was $3.6 \pm 0.7 \mathrm{~min}$ when increasing glucose concentrations, and $12.8 \pm 1.4 \mathrm{~min}$ for the decreasing case.

The longer time constant related to decreasing glucose concentrations could be explained by a smaller mobility of glucose molecules in the viscous sensitive fluid with respect to the isotonic saline solution. This smaller mobility would increase the time required for glucose molecules to find a pore, consequently slowing down the diffusion process when glucose diffuses out of the sensor. Therefore, we expect that the porosity of the semi-permeable membrane affects more the glucose molecules leaving the sensor than glucose molecules entering the sensor.

The response time achieved by our device was longer than our targeted $10 \mathrm{~min}$ response time required for a medical device. This response time may be shortened by reducing the sensor thickness since this would decrease the length for molecules to diffuse, and therefore would decrease the diffusion time. The relaxation time for diffusion is given by $\tau \propto L^{2}$, where $L$ is the characteristic length. According to the above relation, a cavity thickness of $150 \mu \mathrm{m}$ should achieve a response time less than $10 \mathrm{~min}$. Such a dimension is quite standard in microengineering technologies.

\subsection{Glucose sensor reproducibility and stability}

For assessing reproducibility and stability, the sensor was tested in the most relevant physiologically glucose concentrations, namely $2 \mathrm{mM}$ and $20 \mathrm{mM}$ (Fig. 5). Seventeen full cycles performed without interruption during $70 \mathrm{~h}$ demonstrated a high reversibility and stability of the sensor. The phase shift difference between 2 and $20 \mathrm{mM}$ was constant throughout the measurements. For each equilibrium value of glucose concentration, 400 measurements were averaged, leading to a mean standard deviation of $0.045^{\circ}$. The glucose sensitivity was $2.64^{\circ} / \mathrm{mM}$, which combined with a $95 \%$ confidence interval of $0.09^{\circ}(2 \sigma)$, results in an accuracy of $0.034 \mathrm{mM}$. This value is comparable to the $1 \mathrm{mg} / \mathrm{dl}(0.056 \mathrm{mM})$ resolution of conventional glucose meters. In addition, when compared to a reference method, most of the glucose meters have an accuracy characterized by $\sim 95 \%$ of the readings in a $\pm 20 \%$ deviation limit (Brunner et al., 1998). The sensor accuracy corresponds to a relative deviation of $\pm 1.7 \%$ for a low hypoglycaemic value $(2 \mathrm{mM})$, which confirms that the sensor resolution is accurate enough for patients monitoring. Moreover, no decrease in the sensor sensitivity was observed, demonstrating a high stability.

Concerning sensor to sensor variability, this is also an important aspect when moving towards large scale applications, but it can hardly be addressed in this proof of concept study.

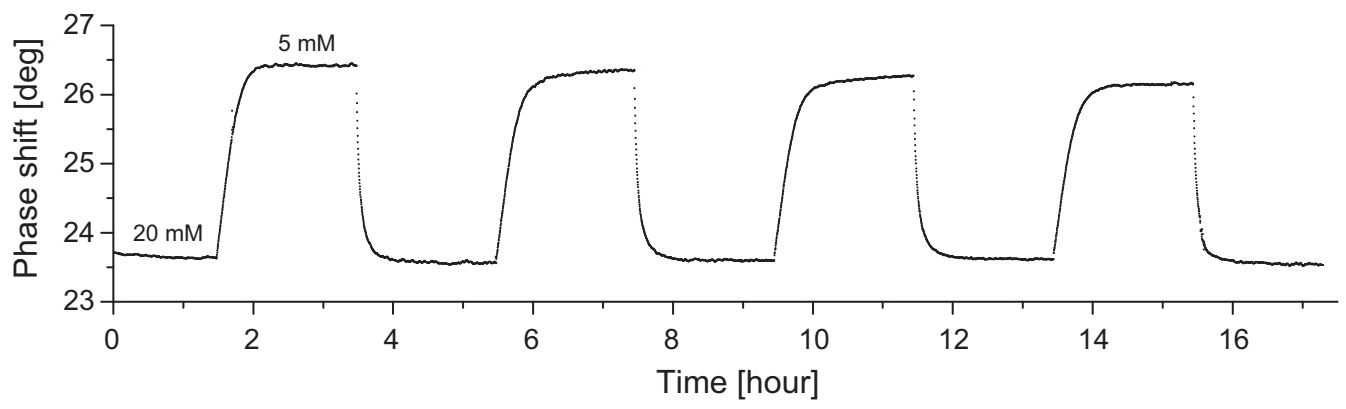

Fig. 6. In vitro determination of glucose in human blood serum. Measurements carried out in original $(5 \mathrm{mM})$ and glucose added $(20 \mathrm{mM})$ serum at $25^{\circ} \mathrm{C}$. 


\subsection{In vitro determination of glucose in human serum}

Although the specificity of ConA for glucose was demonstrated in physiological solutions, once in the blood, some components could interfere with the sensitive solutions. Moreover, $\mathrm{Mn}^{2+}$ which is used to activate ConA, is almost absent in blood, which could affect the stability of ConA. Biofouling of the alumina nanoporous membrane could also be another issue when working in biological fluids. Checking the adequacy of our sensor for in vitro measurements is therefore not trivial.

Therefore, we also tested the sensor in human serum with glucose concentrations of $5 \mathrm{mM}$ and $20 \mathrm{mM}$ (Fig. 6). Nine changes of low and high glucose concentrations were performed during $17 \mathrm{~h}$. A good stability of the sensor response was observed, meaning also that ConA remains chemically stable in presence of serum, which indicates that the lack of $\mathrm{Mn}^{2+}$ does not affect the ConA activation state. Moreover, the response time did not increase with time, indicating that biofouling due to protein adsorption on the semi-permeable membrane, if any, was not an issue and that alumina nanoporous membranes are well-suited for biosensors size-selective interface in biological fluids.

From these measurements, it comes out that the sensor sensitivity in serum is decreased by about $20 \%$ compared to the sensitivity in isotonic saline solution. After evaluation of the glucose in serum, the sensor partly recovers its initial sensitivity for isotonic saline solution. Therefore the loss of sensitivity is reversible, suggesting that small biological molecules, most likely glycosylated peptides or lipids, may diffuse into the sensor and compete with the binding of glucose to ConA. These experiments confirm that despite a slight loss in sensitivity, the sensitivity of the sensor in serum is still high enough for glucose monitoring and the combination of the ConA-based sensitive fluid with the microviscometer is a promising approach for continuous glucose monitoring in blood.

\section{Conclusion}

A viscosity-based affinity sensor for continuous monitoring of glucose in biological fluids was developed using Concanavalin A in dextran as sensing fluid. The sensor was extensively tested in isotonic saline solution and in human blood serum for physiological blood glucose concentrations between 2 and $20 \mathrm{mM}$. The quite high accuracy of $\pm 1.7 \%$ in the hypoglycaemic range demonstrates the ability of this sensor for accurate glucose monitoring. A good degree of reversibility and stability of the sensor was also demonstrated for up to 3 days. Concerning its time response, we report a value of $12.8 \mathrm{~min}$ at $37^{\circ} \mathrm{C}$, which in line with the $10 \mathrm{~min}$ required for medical applications. These results suggest that the combination of the ConA-based sensitive fluid and the microviscometer is a promising sensing principle for continuous glucose monitoring in blood. Further work could include testing in human blood serum at physiological temperature. Using new technological developments, the sensor to sensor variability could also be addressed.

\section{References}

Ballerstadt, R., Ehwald, R., 1994. Biosens. Bioelectron. 9, 557-567.

Ballerstadt, R., Evans, C., Gowda, A., McNichols, R., 2006. Diabetes Technol. Ther. 8, 296-311.

Ballerstadt, R., Kholodnykh, A., Evans, C., Boretsky, A., Motamedi, M., Gowda, A., McNichols, R., 2007. Anal. Chem. 79, 6965-6974.

Ballerstadt, R., Polak, A., Beuhler, A., Frye, J., 2004. Biosens. Bioelectron. 19, 905-914

Ballerstadt, R., Schultz, J.S., 2000. Anal. Chem. 72, 4185-4192.

Beyer, U., Schafer, D., Thomas, A., Aulich, H., Haueter, U., Reihl, B., Ehwald, R., 2001 Diabetologia 44, 416-423.

Brunner, G.A., Ellmerer, M., Sendlhofer, G., Wutte, A., Trajanoski, Z., Schaupp, L., Quehenberger, F., Wach, P., Krejs, G.J., Pieber, T.R., 1998. Diabetes Care 21 585-590.

Diem, P., Kalt, L., Haueter, U., Krinelke, L., Fajfr, R., Reihl, B., Beyer, U., 2004. Diabetes Technol. Ther. 6, 790-799.

Deshpande, M., Saggere, L., 2007. Sens. Actuators A: Phys. 136, 673-689.

Heller, A., Feldman, B., 2008. Chem. Rev. 108, 2482-2505.

Huang, X., Li, S., Schultz, J.S., Wang, Q., Lin, Q., 2009. Sens. Actuators B: Chem. 140 , 603-609.

Klonoff, D.C., 2005. Diabetes Care 28, 1231-1239.

Krinsley, J.S., 2004. Mayo Clin. Proc. 79, 992-1000.

Kuenzi, S., Meurville, E., Ryser, P., 2010. Sens. Actuators B: Chem. 146, 1-7.

Kuenzi, S., Meurville, E., Grandjean, E., Straessler, S., Ryser, P., 2011. Sens. Actuators B: Chem. 167, 194-203.

Schultz, J.S., Mansouri, S., Goldstein, I.J., 1982. Diabetes Care 5, 245-253.

Tierney, S., Falch, B., Hjelme, D., Stokke, B., 2009a. Anal. Chem. 81, 3630-3636.

Tierney, S., Volden, S., Stokke, B.T., 2009b. Biosens. Bioelectron. 24, 2034-2039.

Uchida, S., 1956. ZAMP 7, 403-422.

Wang, J., 2008. Chem. Rev. 108, 814-825.

Zhao, Y., Li, S., Davidson, A., Yang, B., Wang, Q., Lin, Q., 2007. J. Micromech. Microeng. $17,2528-2537$. 